\title{
Behavioral fever response in Rhodnius prolixus (Reduviidae: Triatominae) to intracoelomic inoculation of Trypanosoma cruzi
}

\author{
Gloria Hinestroza ${ }^{[1]}$, Mario Iván Ortiz ${ }^{[2]}$ and Jorge Molina ${ }^{[2]}$
}

[1]. Proyecto Maricultura Isla de Providencia, San Andrés y Providencia, Colombia. [2]. Centro de Investigaciones en Microbiología Parasitología Tropical, Universidad de los Andes, Bogotá, Colombia.

\begin{abstract}
Introduction: Behavioral fever is a response to infections with microorganisms observed in some poikilothermic animals. Rhodnius prolixus is involved in the transmission of two parasites: Trypanosoma cruzi (pathogenic for humans and transmitted in feces) and Trypanosoma rangeli (non-pathogenic for humans, pathogenic for Rhodnius and transmitted by the bite of an infected individual). Only T. rangeli is found in the hemolymph of Rhodnius as it travels to the salivary glands. Methods: To study vector-parasite interactions, we evaluated possible behavioral fever responses of $R$. prolixus to intracoelomic inoculation with T. cruzi or T. rangeli. Temperature preferences of fifth-instar nymphs of $R$. prolixus were evaluated after inoculation with $T$. rangeli $\mathrm{KP} 1(+), \mathrm{KP} 1(-)$, T. cruzi I, or the Trypanosome culture medium. Four different fixed temperatures $\left(25,30,35\right.$, and $\left.40^{\circ} \mathrm{C}\right)$ in two simultaneous experiments (enclosed and free-moving insects) were evaluated. Free-moving insects were marked daily according to their temperature preferences on each of the 15 days after inoculation. Numbers of insects in each temperature shelter and daily mortality were compared with those enclosed shelters of different temperatures. Results: Rhodnius prolixus inoculated with both strains of $T$. rangeli and with the trypanosome culture medium showed preferences for the lowest temperatures $\left(25^{\circ} \mathrm{C}\right)$. However, R. prolixus inoculated with $T$. cruzi I showed significant preferences for temperatures around $35^{\circ} \mathrm{C}$. Conclusions: This is the first known investigation to demonstrate a behavioral fever response in R. prolixus injected intracoelomically with $T$. cruzi I.
\end{abstract}

Keywords: American trypanosomiasis. Rhodnius prolixus. Trypanosoma rangeli. Trypanosoma cruzi. Behavioral fever.

\section{INTRODUCTION}

In endothermic animals, fever is a common internal response to overcome microorganism infections ${ }^{(1)}$. However, ectothermic animals can only raise their internal body temperature to reduce microorganism infections by moving to warmer areas in a classic behavioral response called behavioral fever ${ }^{(1)(2)(3)}$. Evidence that insects can become feverish was first observed in the cockroach Gromphadorhina portentosa injected with an Escherichia coli suspension ${ }^{(4)}$. Studies over the past 25 years ${ }^{(5)(6)(7)(8)(9)}$ have shown that a febrile response is not only possible, but widespread among ectotherms ${ }^{(10)}$. However, to our knowledge, there is only one previous study testing behavioral fever in blood-sucking insects involved in parasite transmissions ${ }^{(11)}$.

Triatominae (Hemiptera: Reduviidae) are blood-sucking insects that are important vectors in the transmission of Trypanosoma cruzi, the causative agent of American

Corresponding author: Dr. rer. nat. Jorge Molina.

e-mail: jmolina@uniandes.edu.co

Received 5 January 2016

Accepted 12 July 2016 trypanosomiasis or Chagas disease ${ }^{(12)}$. Chagas disease is a very complex anthropozoonosis that is widely distributed in 21 LatinAmerican countries, where it affects approximately 7.7 million people, with approximately 12,500 deaths and approximately 41,200 new cases each year ${ }^{(13)}$. In addition, Trypanosoma rangeli (apathogenic to vertebrate hosts) is also frequently found and transmitted by triatomines in Latin-America ${ }^{(14)}$. Interestingly, while $T$. cruzi is apathogenic for triatomines, T. rangeli is considered pathogenic to these insects ${ }^{(15)}$.

In triatomines, $T$. cruzi never leaves the digestive tract system and is transmitted through contaminated feces ${ }^{(16)}$. Conversely, T. rangeli always leaves the digestive tract to travel to the salivary glands and is transmitted through the bite of an infected individual ${ }^{(14)(17)}$. According to Zingales et al. ${ }^{(18)}$, six groups of T. cruzi are recognized (T. cruzi I to VI), while for T. rangeli only two groups have been defined [T. rangeli KP1(-) and T. rangeli $\mathrm{KP} 1(+)]^{(19)}$.

In Colombia, the main vector of Chagas disease is Rhodnius prolixus and T. cruzi I and T. rangeli $\mathrm{KP} 1(+)$ and $\mathrm{KP} 1(-)$ are widely distributed ${ }^{(19)}(20)$. A close association of R. prolixus with $T$. rangeli $\mathrm{KP} 1(+)$ has been observed, as it travels to the salivary glands through the hemolymph. In contrast, $T$. rangeli KP1(-) has been observed to be more closely associated with 
Rhodnius belonging to the pallescens group ${ }^{(17)(21)}$. Recently, a trypanolytic factor acting against $T$. rangeli $\mathrm{KP} 1(-)$ was found in the hemolymph of $R$. prolixus ${ }^{(17)(22)}$.

Thermopreferences in triatomines, such as Rhodnius, have been studied in several species, and are a dynamic process influenced by daily rhythms and starvation levels ${ }^{(23)(24)(25)(26)(27)}$. In Triatoma infestans and T. brasiliensis, daily temperature preferences vary across a broad range ${ }^{(23)(25)}$, while in Panstrongylus megistus and $R$. prolixus, the range of daily temperature preferences varies only slightly ${ }^{(24)(27)}$. Lower temperature preferences in triatomines during the photophase and with increasing starvation would avoid unnecessary waste of water and nutrients ${ }^{(24)}$.

The thermopreferences of Triatomines and the interaction between two Trypanosoma species with different developmental cycles inside Rhodnius represents a model to test the behavioral fever response against microorganisms present in the hemolymph of $R$. prolixus. We intracoelomically inoculated T. cruzi (not present in the hemolymph) and T. rangeli (present in the hemolymph). Our objectives were: 1) To test whether $R$. prolixus is able to adapt thermopreferences to develop a behavioral fever strategy; 2) to test whether behavioral fever depends on specific associations with microorganisms and the body parts they occupy in $R$. prolixus, and 3 ) to analyze the impact of environmental temperature on the survival of intracoelomically infected $R$. prolixus to evaluate the adaptive value of behavioral fever.

\section{METHODS}

\section{Insects}

Rhodnius prolixus were captured from wild populations in 1979 and have been maintained since as a laboratory colony. We used 1,800 fifth-instar nymphs nourished with live hen's blood every 15 days and reared at $27 \pm 2^{\circ} \mathrm{C}, 75 \pm 10 \%$ relative humidity, under a 12/12h light/dark schedule. Nymphs were separated into five treatments for the experiments.

\section{Strains of Trypanosoma}

Three strains of trypanosomes were evaluated in the experiment: a) T. rangeli $\mathrm{KP} 1(+)$, isolated from domestic $R$. prolixus and maintained for 21 cycles in the laboratory; $\mathrm{b}$ ) $T$. rangeli $\mathrm{KP} 1(-)$, isolated from wild $R$. prolixus and maintained for 23 cycles in the laboratory; and c) T. cruzi I, isolated from wild $R$. prolixus and maintained for 20 cycles in the laboratory. All strains were maintained in Tobie culture medium ${ }^{(28)}$ under the same laboratory conditions (temperature $25^{\circ} \mathrm{C}$ and $70 \%$ relative humidity).

\section{Treatments}

Five treatments were evaluated daily for 15 days: Control 1: Non-inoculated insects, Control 2: Insects inoculated only with Tobie culture medium, Experiment 1: T. cruzi I in Tobie culture medium, Experiment 2: T. rangeli $\mathrm{KP} 1(+)$ in Tobie culture medium, and Experiment 3: T. rangeli KP1(-) in Tobie culture medium.

For each treatment including controls, 360 nymphs were evaluated. Nymphs used in control 2 and experiment 1 to
3 were held ventral side up on a flat surface and inoculated intracoelomically between the last fourth abdominal sternites with a tuberculine syringe. Each insect was inoculated with approximately $10 \mu 1$ of Tobie culture medium with a concentration of $1.32 \times 10^{4}$ trypanosomes/ $\mu 1$ (determined with a Neubauer chamber or hemocytometer).

\section{Experimental arena and shelters}

All treatments were conducted on a square slide of superboard $(1 \mathrm{~m} \times 1 \mathrm{~m} \times 2 \mathrm{~cm})$ surrounded by transparent acrylic walls $10 \mathrm{~cm}$ in height and covered with vaseline to prevent the insects from climbing out. A square $(1 \mathrm{~m} \times 1 \mathrm{~m} \times 3 \mathrm{~mm})$ of transparent acrylic covering the experimental arena was used to maintain constant temperature and relative humidity during the experiments. On the experimental arena, four metallic plates $(20 \mathrm{~cm} \times 20 \mathrm{~cm})$ were placed at equal distances from the walls and from each other. Electric heaters were placed below each metallic plate and connected to power supplies through separately controlled thermostats to supply constant and independent temperature to each plate. Experimental temperatures were $25^{\circ} \mathrm{C}, 30^{\circ} \mathrm{C}, 35^{\circ} \mathrm{C}$, and $40^{\circ} \mathrm{C}$ measured with thermometers $\left(-10\right.$ to $\left.80^{\circ} \mathrm{C} \pm 0.1\right)$. A constant light source (Philips Genie $14 \mathrm{~W}$ warm white) in the center of the arena was placed $9 \mathrm{~cm}$ from the acrylic cover to provide $24 \mathrm{~h}$ illumination to the arena (Figure 1). The light source induced insects to search for a shelter to avoid lights ${ }^{(29)}$.

Two rectangular $(10 \mathrm{~cm} \times 20 \mathrm{~cm} \times 5 \mathrm{~cm})$ and unconnected shelters were placed on each of the thermal plates. In total, eight shelters were provided for each treatment, four (shelters A to $D$ in Figure 1) maintained 60 nymphs each at a constant temperature, while the other four (shelters $\mathrm{E}$ to $\mathrm{H}$ in Figure 1) were open to both lateral sides and allowed 120 free-moving insects to choose one of the four temperatures available.

The number of free-moving insects inside each shelter was counted daily at 18:00h, and individuals were marked on the legs according to their preferred temperature.

\section{Dissections and parasitemia}

Daily dissections of two insects from each of the closed shelters (A to D Figure 1) were conducted at $24 \mathrm{~h}$ postinoculation. Salivary glands of nymphs infected with $T$. rangeli KP1(+), salivary glands and hindguts (rectal ampulla) of $R$. prolixus infected with $T$. rangeli $\mathrm{KP} 1(-)$, and hindguts (rectal ampulla) of $R$. prolixus infected with $T$. cruzi I were dissected in Phosphate Buffered Solution $\mathrm{pH}=7.2$ according to standard methodology ${ }^{(30)}$. Average numbers of parasites were determined daily with a Neubauer chamber or hemocytometer using a light microscope at $400 \times$ magnification.

\section{Data analysis}

The effect of treatments and temperatures on mortality of insects enclosed in shelters A to D and the average number of insects in shelters $\mathrm{E}$ to $\mathrm{H}$ after 15 days post-inoculation were analyzed with two-way analysis of variance (ANOVA), to determine the effect of each variable independently, and the interaction between them. We assumed a mixed-design ANOVA model with one fixed effect factor (treatment: T. cruzi or T. rangeli inoculation) and one random effect factor (temperature). 


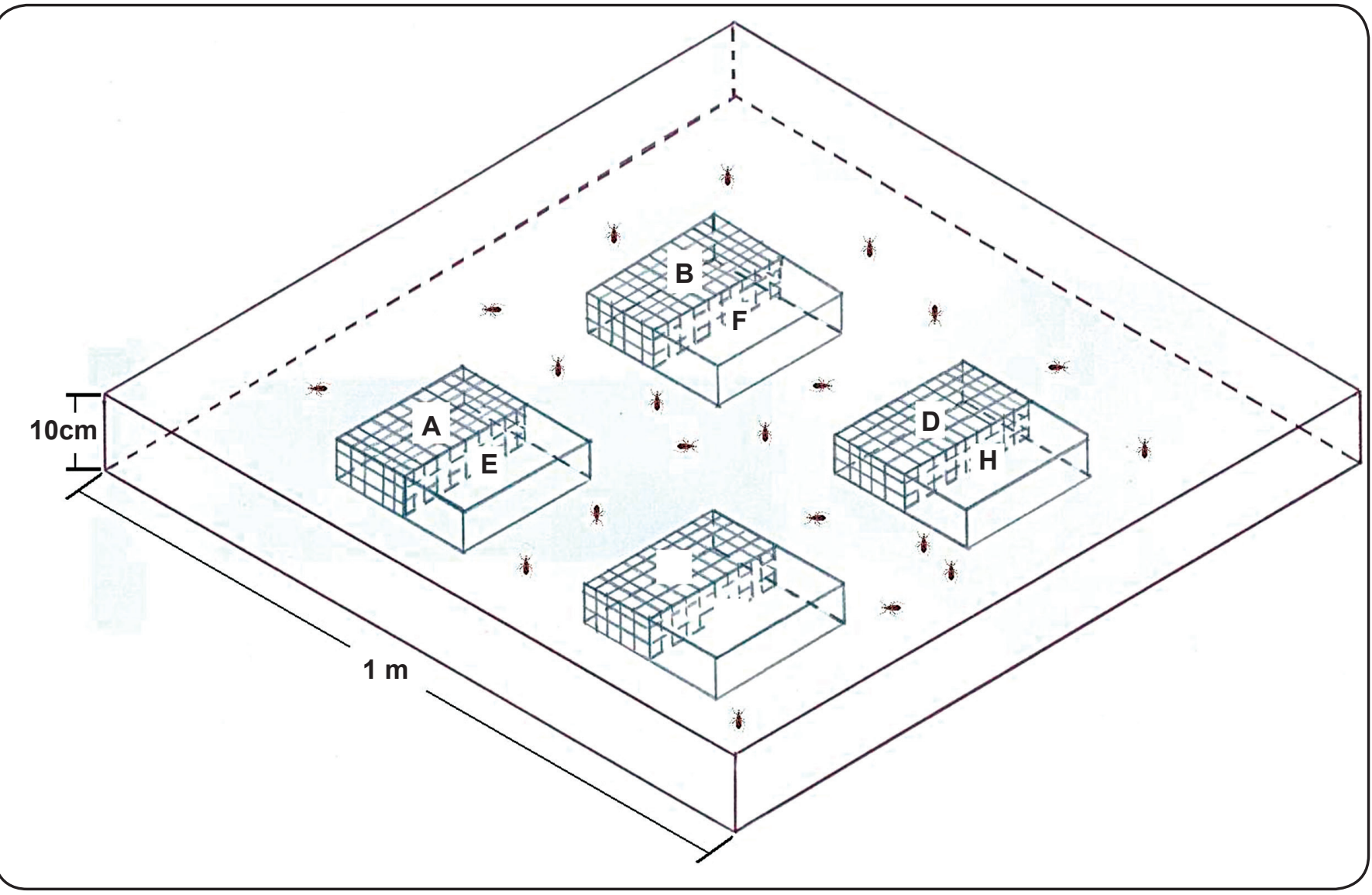

FIGURE 1. Experimental arena with shelters. Shelters A to D: housed insects continually enclosed at a constant temperature. Shelters E to H: were open and free to access by free-moving fifth-instar nymphs of Rhodnius prolixus

Post-hoc multiple comparisons using the Scheffé test were conducted to evaluate the effect of treatments and temperature on the survival of $R$. prolixus $^{(31)}$. All statistical analyses were performed with the software Statgraphics Centurion XVI.

\section{RESULTS}

Rhodnius prolixus maintained at constant temperatures for 15 days (shelters A to D in Figure 1) only showed the presence of $T$. rangeli $\mathrm{KP} 1(+)$ in salivary glands after the third day post-inoculation (Figure 2). The average number of parasites detected in salivary glands increased until ten days postinoculation and was higher in insects maintained in shelters at $25^{\circ} \mathrm{C}$ (Figure 2). No trypanosomes were found in the salivary glands or rectal ampullae of insects inoculated with $T$. rangeli KP1(-) or T. cruzi I.

Rhodnius prolixus kept at constant temperatures for 15 days (shelters A to D in Figure 1) showed statistically significant differences in mortality between the five treatments tested $(\mathrm{p}=0.015, \mathrm{~F}$-ratio $=7.4, \mathrm{df}=4$ two-way ANOVA $)$, between the temperatures tested $(\mathrm{p}=0.028, \mathrm{~F}$-ratio $=5.2, \mathrm{df}=3$ two-way ANOVA), and a significant interaction between treatments and temperatures $(\mathrm{p}=0.038, \mathrm{~F}$-ratio $=4.2, \mathrm{df}=1$ two-way ANOVA $)$. Post-hoc analysis showed only statistical significances in mortalities of control $R$. prolixus (non-inoculated or inoculated with Tobie medium) between the lower temperatures ( 25 and $\left.30^{\circ} \mathrm{C}\right)$ and higher temperatures $\left(35\right.$ and $\left.40^{\circ} \mathrm{C}\right)(\mathrm{p}<0.05$ Scheffé test). Insects inoculated with $T$. rangeli $\mathrm{KP} 1(-)$ showed statistical differences only between $35^{\circ} \mathrm{C}$ and the other temperatures tested $(\mathrm{p}<0.03$ Scheffé test), and insects inoculated with T. cruzi showed statistical differences in mortality between all temperatures tested, except when comparing 30 and $40^{\circ} \mathrm{C}$ $(\mathrm{p}<0.05$ Scheffé test). No significant differences were observed between the temperatures in insects inoculated with $T$. rangeli $\mathrm{KP} 1(+)(p>0.05$ Scheffé test $)$. All together, these results confirm that mortalities higher than 10 insects found in both controls at $25^{\circ} \mathrm{C}$ and $30^{\circ} \mathrm{C}$ is suggesting that factors additional to hunger and inoculation procedures may have caused mortality of the triatomines (Figure 3).

Two interactive effects of temperature and treatment on results considering mortality are of especial interest: a reduction in mortality in R. prolixus inoculated with $T$. cruzi and T. rangeli $\mathrm{KP} 1(-)$ at $35^{\circ} \mathrm{C}(\mathrm{p}<0.05$ Scheffé test), and, an increase in mortality at $35^{\circ} \mathrm{C}$ in insects inoculated with $T$. rangeli $\mathrm{KP} 1(+)$ compared to the control $(\mathrm{p}<0.05$ Scheffé test). This suggested that $R$. prolixus maintained at $35^{\circ} \mathrm{C}$ were possibly able to control a parasite infection, with the exception of those inoculated with T. rangeli $\mathrm{KP} 1(+)$ (Figure 3). A temperature of $40^{\circ} \mathrm{C}$ still had some effect on parasites; however, it appears that the effect of 


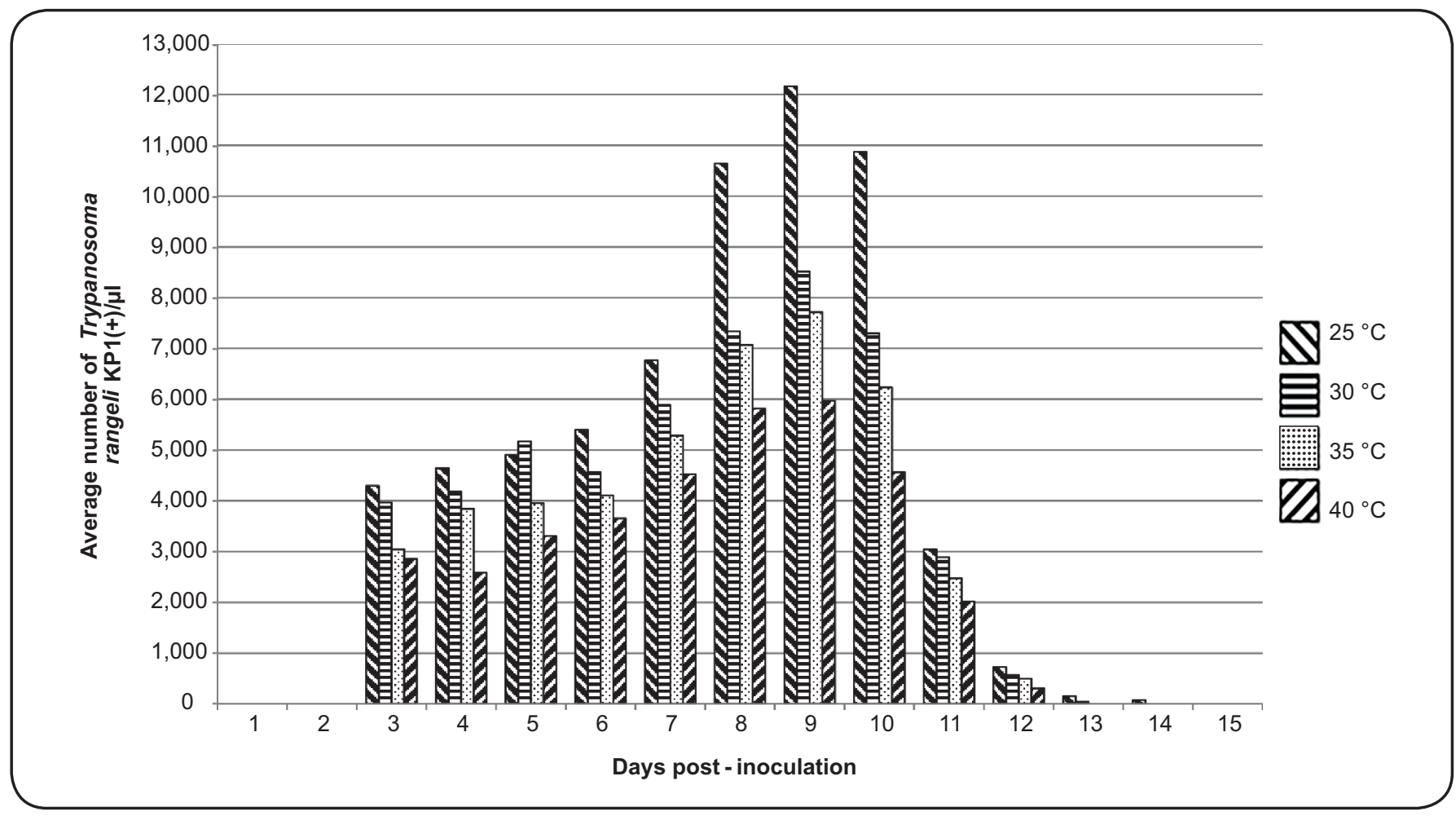

FIGURE 2. Average number of Trypanosome rangeli KP1(+) counted daily in salivary glands of Rhodnius prolixus maintained at a constant temperature for 15 days.

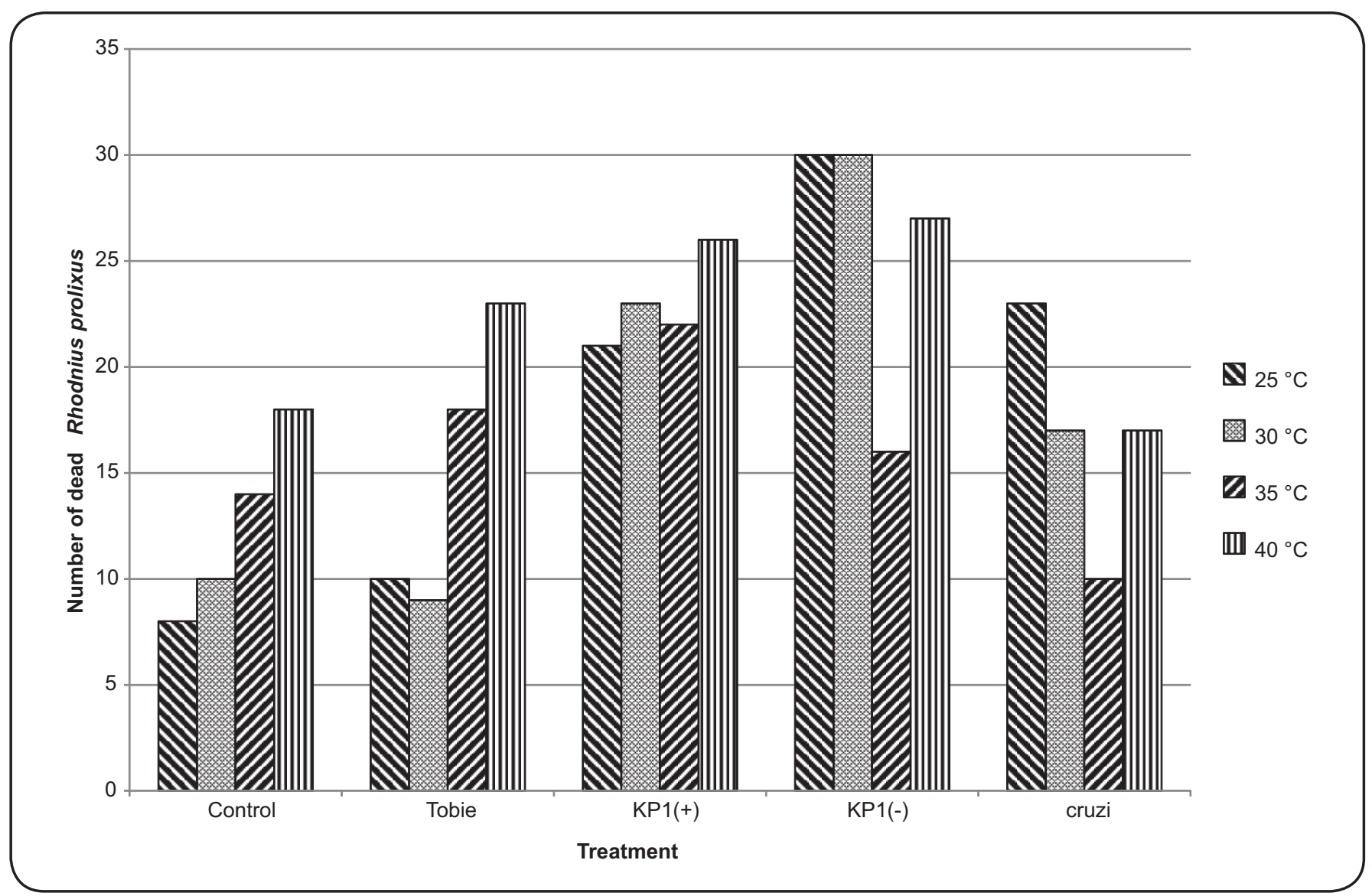

FIGURE 3. Total number of dead Rhodnius prolixus after 15 days maintained at a constant temperature. 


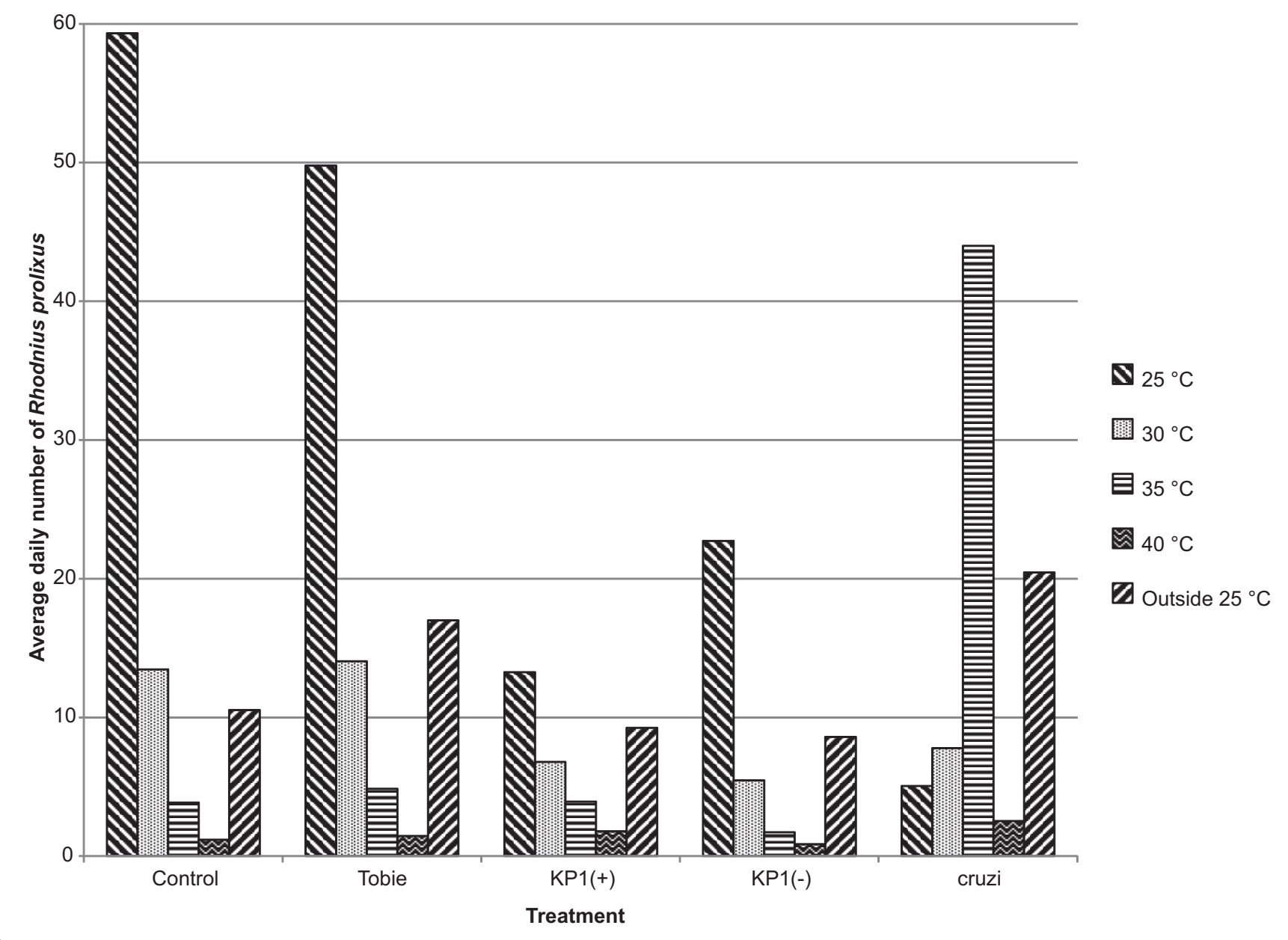

FIGURE 4. Average daily number of living Rhodnius prolixus observed over the 15 days in each evaluated temperature.

temperature on the insects was stronger and again, an increase in mortality was observed in all treatments (Figure 3).

These results indicated that Rhodnius prolixus inoculated only with $T$. rangeli KP1(-) or T. cruzi and that were free to move between shelters with different temperatures searched for shelters with temperatures around $35^{\circ} \mathrm{C}$ to overcome the infection.

Interestingly, insects that were free to move between shelters (E to $\mathrm{H}$ in Figure 1) showed preferences related to treatment $(\mathrm{p}=0.04, \mathrm{~F}$-ratio $=4.1, \mathrm{df}=4$ two-way ANOVA $)$, temperature $(\mathrm{p}=0.02$, F-ratio $=5.8, \mathrm{df}=3$ two-way ANOVA $)$, and a significant interaction between both factors $(\mathrm{p}=0.042$, F-ratio $=4.0, \mathrm{df}=1$ two-way ANOVA) (Figure 4). Post-hoc analysis showed statistical significant preferences in R. prolixus in control experiments (non-inoculated or inoculated with Tobie medium) for shelters at $25^{\circ} \mathrm{C}(\mathrm{p}<0.02$ Scheffé test $), 30^{\circ} \mathrm{C}$, or $25^{\circ} \mathrm{C}$ outside ( $\mathrm{p}<0.05$ Scheffé test), and that insects consistently avoided shelters at $35^{\circ} \mathrm{C}$ or $40{ }^{\circ} \mathrm{C}$ (Figure 4). Insects inoculated with T. rangeli $\mathrm{KP} 1(+)$ or $\mathrm{KP} 1(-)$ also showed a preference for shelters at $25^{\circ} \mathrm{C}(\mathrm{p}<0.05$ Scheffé test $)$; however, on average more nymphs inoculated with $T$. cruzi were found in shelters at $35^{\circ} \mathrm{C}(\mathrm{p}<0.03$ Scheffé test) or at $25^{\circ} \mathrm{C}$ outside ( $\mathrm{p}<0.04$ Scheffé test) (Figure 4).
Finally, a post-hoc comparison of treatments and temperatures showed that $R$. prolixus inoculated with $T$. cruzi significantly preferred shelters at $35^{\circ} \mathrm{C}$ in comparison to all other treatments at the same temperature $(\mathrm{p}<0.05$ Scheffé test).

In summary, $R$. prolixus inoculated with $T$. rangeli $[\mathrm{KP} 1(+)$ or KP1(-)] showed higher mortality rates and resulted in fewer live insects after 15 days on average, compared to non-inoculated insects, and insects inoculated with Tobie or with T. cruzi (Figure 4).

\section{DIsCussION}

Insects can respond to microbial and parasite infections by altering their thermoregulatory behavior and developing behavioral fever. An increase in body temperature above normal thermal levels from moving to warmer areas has been shown to favor host survival in a number of host-pathogen interactions $^{(4)(5)(6)(7)(8)(9)}$, including Rhodnius prolixus-Trypanosoma interactions (Figure 3 and Figure 4).

Thomas et al. ${ }^{(11)}$ demonstrated behavioral fever in Xenopsylla cheopis infected with Yersinia pestis. Results showed that fleas infected with Yersinia bacteria did not exhibit preferences for high temperatures. 
Triatominae insects exposed to constant light sources exhibit a strong, negative, phototactic response ${ }^{(29)}$. Our experiments used this phototactic response to induce infected Rhodnius prolixus to move to one of the four dark shelters with different temperatures available in the experimental arena.

We hypothesized that $R$. prolixus inoculated with $T$. rangeli $\mathrm{KP} 1(+)$ and $T$. rangeli KP1(-) should behaviorally search for shelters with higher temperatures to overcome the infection with parasites that are pathogenic for triatomines ${ }^{(14)(15)}$. However, our experiments with nymphs maintained at constant temperatures showed that non-inoculated triatomines or insects inoculated with Tobie presented a higher mortality at $35^{\circ} \mathrm{C}$ and $40^{\circ} \mathrm{C}$ (Figure 3). Therefore, individuals must achieve a balance between temperatures high enough to reduce the infection, but not survival.

Trypanosoma cruzi and T. rangeli in Tobie culture medium under laboratory conditions are incubated at $25^{\circ} \mathrm{C}$ to $28^{\circ} \mathrm{C}^{(32)}$. Constant temperature above $30^{\circ} \mathrm{C}$ is lethal for trypanosomes in culture medium (unpublished data from our group), suggesting that shelters at $35^{\circ} \mathrm{C}$ could provide the ideal solution to reduce infection with minimum impact on survival.

The interaction between temperature and treatments presented two possible results depending on the parasite that was inoculated: a synergistic effect between temperature and parasites (in this case an increase in mortality was expected in triatomines exposed to higher temperatures compared to those exposed to lower temperatures); and, an inhibitory effect between temperature and parasites (in this case lower mortality was expected in triatomines exposed to higher temperatures than in those exposed to lower temperatures). We regarded the second possibility as the true behavioral fever response.

Our results showed both kinds of possible responses (Figure 3). Rhodnius prolixus showed an increase in mortality when T. rangeli $\mathrm{KP} 1(+)$ and higher temperature were positively associated (See Figure 3). Conversely, a reduction in mortality of $R$. prolixus was observed when insects were inoculated with T. rangeli $\mathrm{KP} 1(-)$ or $T$. cruzi exclusively at $35^{\circ} \mathrm{C}$ (Figure 3).

The differences in responses observed between $R$. prolixus inoculated with $T$. rangeli $\mathrm{KP} 1(+)$ and $\mathrm{KP} 1(-)$ can be partially explained by coevolutionary processes. $T$. rangeli $\mathrm{KP} 1(-)$ is a trypanosome circulating in the Andes mountains and is mainly associated with Rhodnius of the pallescens group, while T. rangeli $\mathrm{KP} 1(+)$ circulates to the east of the Andes in close association with Rhodnius of the prolixus group ${ }^{(21)(33)}$.

Given the results from $R$. prolixus maintained at a constant temperature, we expected that bugs free to move to a shelter would prefer $35^{\circ} \mathrm{C}$, but only if they were inoculated with T. rangeli $\mathrm{KP} 1(-)$ or $T$. cruzi.

Experiments with free-moving triatomines showed preferences for shelters at 25 and $30^{\circ} \mathrm{C}$ in both non-inoculated $R$. prolixus and those inoculated with Tobie medium (Figure 4). These results were unsurprising as this is the temperature range maintained in the laboratory breeding conditions and corresponds to the temperatures recommended for triatomines care and maintenance procedures ${ }^{(34)}$.
However, when nymphs of $R$. prolixus were inoculated with T. cruzi they showed behavioral fever responses by moving to shelters at $35^{\circ} \mathrm{C}$ (Figure 4).

A possible explanation for the behavioral fever response observed in $R$. prolixus could be the route of inoculation. Intracoelomic inoculation introduces T. cruzi (a non-pathogenic Trypanosoma for triatomines) directly into the hemocoel, where T. cruzi is not present under natural conditions ${ }^{(35)}$. In other words, the presence of a foreign cell in the hemocoel produced a behavioral fever response to overcome the infection and reduce mortality (Figure 3).

Why was behavioral fever not observed in $R$. prolixus inoculated with $T$. rangeli $\mathrm{KP} 1(-)$ ? A possible explanation is a recently discovered trypanolytic protein in $R$. prolixus that can function as an additional barrier against parasites. This molecule is responsible for inhibiting the development of $T$. rangeli $\mathrm{KP} 1(-)$, making $R$. prolixus resistant to this kind of parasite $\operatorname{strain}^{(17)}$. In addition, it has been demonstrated that behavioral fever in insects is a specific response expressed toward a small subset of pathogens ${ }^{(8)}$.

From an immunological point of view, behavioral fever responses to a subset of pathogens can be expected as different survival of T. rangeli and T. cruzi in the hemolymph of R. prolixus has been observed after inoculation into the hemocoel ${ }^{(35)(36)}$. Differences in the survival of parasites have been related to an induction of lysozymes, agglutinating, prophenoloxidase, and hemocyte activity ${ }^{(36)(37)}$. For instance, the presence of $T$. rangeli in the hemocoel of $R$. prolixus activates the prophenoloxidase system, phagocytosis, hemocyte microaggregation, superoxide and nitric oxide activity, and eicosanoid biosynthesis ${ }^{(38)}$.

This study of $R$. prolixus inoculated intracoelomically with Trypanosomes showed that behavioral fever should only be expected in those cases where hosts have not coevolved to make the appropriate response against parasites. As with other insects $^{(4)(5)(6)(7)(8)(9)}$, R. prolixus is only able to develop behavioral fever against unknown microorganisms in the hemolymph.

Future experiments with other triatomine species, including different routes of infection, such as ingesting blood infected with trypanosomes, would confirm the effect of behavioral fever on Trypanosoma spp. It would also be interesting to evaluate the effect of behavioral fever on natural populations of triatomines exposed to pathogens that are potentially involved in biological control, such as fungi ${ }^{(9)(39)(40)(41)(42)(43)}$ and viruses ${ }^{(44)(45)}$.

\section{Conflict of interest}

The authors declare that there is no conflict of interest.

\section{Acknowledgments}

We would like to thank Dr. Gustavo Vallejo (Laboratorio de Investigaciones en Parasitología Tropical, Universidad del Tolima, Ibagué, Colombia) for supplying the $T$. rangeli $\mathrm{KP} 1(-)$ strain.

\section{Financial support}

This study was supported by the Research Funds of the Faculty of Sciences, Universidad de Los Andes, Bogotá. 


\section{REFERENCES}

1. Casterlin ME, Reynolds WW. Behavioral fever in the crayfish. Hydrobiologia 1977; 56:99-101.

2. Cabanac M, Rossetti Y. Fever in snails, reflection on a negative result. Comp Physiol Biochem 1987; 87A:1017-1020.

3. Sherman E, Baldwin L, Fernandez G, Deurrel E. Fever and thermal tolerance on the toad Bufo marinus. J Therm Biol 1991; 16:297-301.

4. Boorstein SM, Conner WE. Endotoxin-induced behavioral fever in the Madagascar cockroach Gromphadorhina portentosa. J Insect Physiol 1984; 30:327-330.

5. Boorstein SM, Ewald PW. Costs and benefits of behavioral fever in Melanoplus sanguinipes infected by Nosema acridophagus. Physiol Zool 1987; 60:586-595.

6. McClain E, Magnuson P, Warner SJ. Behavioural fever in a namib desert tenebrionid beetle, Onymacris plana. J Insect Physiol 1988; 34:279-284.

7. Watson DW, Mullens BA, Petersen JJ. Behavioral fever response of Musca domestica (Diptera: Muscidae) to infection by Entomophthora muscae (Zygomycetes: Entomophthorales). J Invert Pathol 1993; 61:10-16.

8. Adamo SA. The specificity of behavioral fever in the cricket Acheta domesticus. J Parasitol 1998; 84:529-533.

9. Ouedraogo RM, Cusson M, Goettel MS, Brodeur J. Inhibition of fungal growth in thermoregulating locusts, Locusta migratoria, infected by the fungus Metarhizium anisopliae var acridum. J Invert Pathol 2003; 82:103-109.

10. Moore J. Parasites and the behavior of animals. Oxford: Oxford University Press; 2002.

11. Thomas RE, Karstens RH, Schwan TG. Effect of Yersinia pestis infection on temperature preference and movement of the oriental rat flea (Xenopsylla cheopis) (Siphonaptera: Pulicidae). J Med Entomol 1993; 30:209-213.

12. Lent H, Wygodzinsky P. Revision of the Triatominae (Hemiptera, Reduviidae), and their significance as vectors of Chagas disease. Bull Am Mus Nat Hist 1979; 163:123-520.

13. Organización Mundial de la Salud. Reporte del Grupo de trabajo científico sobre la enfermedad de Chagas, 2005. Ginebra: TDR/ Organización Mundial de la Salud;

14. Guhl F, Vallejo G. Trypanosoma (Herpetosoma) rangeli Tejera, 1920: An updated review. Mem Inst Oswaldo Cruz 2003; 98: 435-442.

15. Schaub GA. Pathogenicity of trypanosomatids on insects. Parasitol Today 1994; 10:463-468.

16. Moncayo A, Ortiz Yanine MI. An update on Chagas disease (human American trypanosomiasis). Ann Trop Med Parasitol 2006; 100:663-677.

17. Pulido XC, Perez G, Vallejo GA. Preliminary characterization of a Rhodnius prolixus hemolymph trypanolytic protein, this being a determinant of Trypanosoma rangeli KP1(+) and KP1(-) subpopulations' vectorial ability. Mem Inst Oswaldo Cruz 2008; 103:172-179.

18. Zingales B, Andrade SG, Briones MRS, Campbell DA, Chiari E, Fernandes O, et al. A new concensus for Trypanosoma cruzi intraspecific nomenclature: Second revision meeting recommends TcI to TcVI. Mem Inst Oswaldo Cruz 2009; 104:1051-1054.

19. Vallejo GA, Guhl F, Carranza JC, Lozano E, Sanchez JL, Jaramillo JC, et al. kDNA markers define two major Trypanosoma rangeli lineages in Latin-America. Acta Trop 2002; 81:77-82.
20. Herrera C, Bargues MD, Fajardo A, Montilla M, Triana O, Vallejo GA, et al. Identifying four Trypanosoma cruzi I isolate haplotypes from different geographic regions in Colombia. Infect Genet Evol 2007; 7:535-539.

21. Vallejo GA, Guhl F, Carranza JC, Triana O, Pérez G, Ortiz PA, et al. Interacción tripanosoma-vector-vertebrado y su relación con la sistemática y la epidemiología de la tripanosomiasis americana. Biomédica 2007; 27:110-118.

22. Sánchez IP, Pulido XC, Carranza JC, Triana O, Vallejo GA. Inmunidad natural de $R$. prolixus (Hemiptera: Reduviidae: Triatominae) frente a la infección con Trypanosoma (Herpetosoma) rangeli KP1(-) aislados de $R$. pallescens, $R$. colombiensis y $R$. ecuadoriensis. Rev Asoc Col Cien Biol 2005; 17:108-118.

23. Lazzari CR. Temperature preference in Triatoma infestans (Hemiptera: Reduviidae). Bull Entomol Res 1991; 81:273-276.

24. Pires HHR, Lazzari CR, Schilman PE, Diotaiuti L, Lorenzo MG. Dynamics of thermopreference in the Chagas disease vector Panstrongylus megistus (Hemiptera: Reduviidae). J Med Entomol 2002; 39:716-719.

25. Guarneri AA, Lazzari C, Xavier AAP, Diotaiuti L, Lorenzo MG. The effect of temperature on the behaviour and development of Triatoma brasiliensis. Physiol Entomol 2003; 28:185-191.

26. Minoli SA, Lazzari CR. Chronobiological basis of thermopreference in the haematophagous bug Triatoma infestans. J Insect Physiol 2003; 49:927-932.

27. Schilman PE, Lazzari CR. Temperature preference in Rhodnius prolixus, effects and possible consequences. Acta Trop 2004; 90:115-122.

28. Vallejo GA, Marinkelle CJ, Guhl F, de Sanchez N. Laboratory maintenance of Trypanosoma (Herpetosoma) rangeli Tejera, 1920. Rev Biol Trop 1986; 34:75-81.

29. Reisenman CE, Lazzari CR, Giurfa M. Circadian control of photonegative sensitivity in the haematophagous bug Triatoma infestans. J Comp Physiol A 1998; 183:533-541.

30. Molyneux DH, Ashford RW. The Biology of Trypanosoma and Leishmania, Parasites of Man and Domestic Animals. London: Taylor and Francis; 1983. 294p.

31. Zar JH. Biostatistical Analisis. New Jersey: Prentice Hall; 1999. $663 p$.

32. Bernabé C, Tibayrenc M. Trypanosoma cruzi: long-term subcultures in two different culture media do not confirm the existence of highly versatile multilocus genotypes. Int J Parasitol 2004; 34:779-784.

33. Urrea DA, Carranza JC, Cuba CA, Gurgel-Goncalves R, Guhl F, Schofield CJ, et al. Molecular characterisation of Trypanosoma rangeli strains isolated from Rhodnius ecuadoriensis in Peru, $R$. colombiensis in Colombia and $R$. pallescens in Panama, supports a co-evolutionary association between parasites and vectors. Infect Genet Evol 2005; 5:123-129.

34. Azambuja P, Garcia ES. Care and maintenance of triatomine colonies. In: Crampton JM, Beard CB, Louis C, editors. The molecular biology of insect disease vectors: a methods manual. London: Chapman \& Hall; 1997. p. 56-64.

35. Mello CB, Azambuja P, Garcia ES, Ratcliffe NA. Differential in vitro and in vivo behavior of three strains of Trypanosoma cruzi in the gut and hemolymph of Rhodnius prolixus. Exp Parasitol 1996; $82: 112-121$

36. Mello CB, Garcia ES, Ratcliffe NA, Azambuja P. Trypanosoma cruzi and Trypanosoma rangeli: Interplay hemolymph components of Rhodnius prolixus. J Invert Pathol 1995; 65:261-268. 
37. Azambuja P, Feder D, Mello CB, Gomes SAO, Garcia ES. Immunity in Rhodnius prolixus: Trypanosomatid-vector interactions. Mem Inst Oswaldo Cruz 1999; 94 (suppl I):219-222.

38. Azambuja P, Ratcliffe NA, Garcia ES. Towards an understanding of the interactions of Trypanosoma cruzi and Trypanosoma rangeli within the reduviid insect host Rhodnius prolixus. An Acad Bras Cienc 2005; 77:397-404.

39. Inglis GD, Johnson DL, Goettel MS. Effects of temperature and thermoregulation on mycosis by Beauveria bassiana in Grasshoppers. Biol Control 1996; 7:131-139.

40. Fargues J, Luz C. Effects of fluctuating moisture and temperature regimes on the infection potential of Beauveria bassiana for Rhodnius prolixus. J Invert Pathol 2000; 75:202-211.

41. Lecuona RE, Rodriguez J, La Rossa FR. Effect of constant and cyclical temperatures on the mortality of Triatoma infestans
(Klug) (Hemiptera:Reduviidae) treated with Beauveria bassiana (Bals.) Vuill. (Hyphomycetes). Neotrop Entomol 2005; 34:675-679.

42. Pedrini N, Mijailovsky SJ, Girotti JR, Stariolo R, Cardozo RM, Gentile A, et al. Control of pyrethroid-resistant Chagas disease vectors with entomopathogenic fungi. PLoS Negl Trop Dis 2009; 3:e434.

43. Anderson RD, Blandford S, Jenkins NE, Thomas MB. Discriminating fever behavior in house flies. PLoS ONE 2013; 8:e62269.

44. Muscio OA, La Torre JL, Bonder MA, Scodeller EA. Triatoma virus pathogenicity in laboratory colonies of Triatoma infestans (Hemiptera: Reduviidae). J Med Entomol 1997; 34:253-256.

45. Rozas-Dennis GS, Cazzaniga NJ. Effects of Triatoma virus (TrV) on the fecundity and moulting of Triatoma infestans (Hemiptera: Reduviidae). Ann Trop Med Parasitol 2000; 94:633-636. 